\title{
THE SPACE OF FUNCTIONS OF BOUNDED VARIATION ON CURVES IN METRIC MEASURE SPACES
}

\author{
O. MARTIO \\ Dedicated to Jan Malý on his $60^{\text {th }}$ birthday
}

\begin{abstract}
The approximation modulus, $A M$-modulus for short, was defined in an earlier paper by the author. In this paper it is shown that an $L^{1}(X)-$ function $u$ in a metric measure space $(X, d, \nu)$ can be defined to be of bounded variation on $A M$-a.e. curve in $X$ without an approximation of $u$ by Lipschitz or Newtonian functions. The essential variation of $u$ on $A M$ almost every curve is bounded by a sequence of non-negative Borel functions in $L^{1}(X)$. The space of such functions is a Banach space and there is a Borel measure associated with $u$. For $X=\mathbf{R}^{n}$ this gives the classical space of BV functions.
\end{abstract}

\section{INTRODUCTION}

Since the pioneering work of Ahlfors and Beurling the modulus method has been widely used in conformal geometry in $\mathbf{R}^{n}, n \geq 2$, and, more recently, in metric measure spaces; see [10,11. Fuglede [8] showed that the method is useful in the theory of function spaces and in the study of regularity properties of functions in these spaces. The modulus method has also been applied to function spaces in metric measure spaces (see [4,5, 15]), where it has a more fundamental role than in $\mathbf{R}^{n}$.

In this paper a new modulus, the $A M$-modulus, is used to construct a function space of functions of bounded variation (BV) in a metric measure space $X$. The construction corresponds to the construction of the so-called Newtonian spaces $N^{1, p}(X)$ in $X$; see [5, 15]. Instead of the concept of a (weak) upper gradient we use the concept of a BV upper bound which consists of a sequence of non-negative Borel functions in $L^{1}(X)$.

An $N^{1, p}(X)$ function need not be absolutely continuous on every curve; it is necessary to omit the family of curves of $M_{p}$-modulus zero. Similarly a BV function need not be of bounded variation on every curve. This is already evident in $\mathbf{R}$ where the function $u=\chi_{\{0\}}$ is not BV on every curve. For example, $u$ is not BV on the curve which crosses 0 infinitely often. The $A M$-modulus takes care of this situation.

It is customary to consider BV functions in $X$ defined only almost everywhere. From the approximation point of view $L^{1}(X)$ is a good choice for the base space and this approach is used both in $\mathbf{R}^{n}$ (see [7]) and in $X$ (see [14]). In [1,2,6] several alternative definitions for BV functions in $X$ based on approximation by Lipschitz functions are discussed. As in the case of the Newtonian spaces our approach does not use approximation by smooth functions. It turns out, as in the Newtonian

Received by the editors September 24, 2015 and, in revised form, February 10, 2016.

2010 Mathematics Subject Classification. Primary 26A45; Secondary 26B30, 30L99.

Key words and phrases. Metric measure space, $A M$-modulus, functions of bounded variation. 
space case, that in $\mathbf{R}^{n}$ our approach and the approximation approach lead to the same space.

The $A M$-modulus can be applied to other base spaces than $L^{1}(X)$ (see 13 , Section 4]), but we consider $L^{1}(X)$ since it naturally leads to a Banach space of BV functions in $X$. The space $L^{1}(X)$ also has the advantage that its functions are well defined on $M_{1}$-a.e. curve and thus on $A M$-a.e. curve.

In a forthcoming paper with V. Honzlová-Exernova and J. Malý we show that there exist curve families $\Gamma$ in $\mathbf{R}^{n}, n \geq 1$, such that $M_{1}(\Gamma)=\infty$ but $A M(\Gamma)=0$. Since $A M(\Gamma) \leq M_{1}(\Gamma)$ for every curve family $\Gamma$ in $X$, this shows that the $A M-$ modulus is essentially weaker than the $M_{1}$-modulus.

After preliminaries in Section 2 we prove the Banach property of the space $B V_{A M}(X)$ of $\mathrm{BV}$ functions in Section 3. Section 4 is devoted to the construction of a Borel measure associated with $u$. The construction resembles the construction due to Miranda in 14 who used approximation by Lipschitz functions with $L^{1}$ bounded upper gradients for $u$. In Section 5 the case $X=\mathbf{R}^{n}$ is considered. We use minimal assumptions on the space $(X, d)$ and the measure $\nu$.

Our notation is standard.

\section{Preliminaries}

We recall the basic properties of functions of bounded variation and the $A M-$ modulus and introduce the concept of a $B V_{A M}$ upper bound for a function $u \in$ $L^{1}(X)$.

Let $(X, d, \nu)$ be a metric measure space where $d$ is a metric and $\nu$ is a regular Borel measure in $X$, We use the definition in [5, I.1.1] for regularity and, in particular, $\nu$ is an outer measure in $X$. In addition we assume that $\nu$ satisfies

$$
0<\nu(B(x, r))<\infty
$$

for each ball $B(x, r)$ in $X$. In Section 4 we make some additional assumptions on the space $(X, d)$.

Functions of bounded variation. Suppose that $u:[a, b] \rightarrow \mathbf{R} \cup\{\infty,-\infty\}$ and there is a set $C \subset[a, b]$ such that $m_{1}([a, b] \backslash C)=0$ and $u \mid C$ is of bounded variation on $C$. The latter means that

$$
V(u, C)=\sup \sum_{i=1}^{n}\left|u\left(x_{i}\right)-u\left(x_{i-1}\right)\right|<\infty
$$

where the supremum is taken over all finite sequences $x_{0}<x_{1}<\cdots<x_{n}$ of points in $C$. Such a function $u$ is said to be of essentially bounded variation in $[a, b]$. Then (see [13, Section 2]), there is a function $\hat{u}:[a, b] \rightarrow \mathbf{R}$ such that $\hat{u}=u$ a.e. in $[a, b]$ and

$$
V(\hat{u},[a, b])=\inf \{V(v,[a, b]): v=u \text { a.e. in }[a, b]\} .
$$

Moreover, the function $\hat{u}$ can be chosen to be right continuous in $[a, b)$ and continuous at $b$. With these properties $\hat{u}$ is uniquely determined by $u$, i.e. if $u=v$ a.e. in $[a, b]$, then $\hat{u}=\hat{v}$. Note also that

$$
V(\hat{u},[a, b])=V(\hat{u}, C)
$$


for every $C \subset[a, b]$ of full measure, since if $V(\hat{u},[a, b])>V(\hat{u}, C)$, then by [13, Lemma 2.1] there is a function $v$ in $[a, b]$ such that $v=\hat{u}$ in $C$ and

$$
V(v,[a, b])=V(v, C)=V(\hat{u}, C)
$$

so that $\hat{u}$ is not of minimal variation.

Lemma 2.1. Suppose that $u_{i} \rightarrow u$ in $L^{1}([a, b])$. Then

$$
V(\hat{u},[a, b]) \leq \liminf _{i \rightarrow \infty} V\left(\hat{u}_{i},[a, b]\right) .
$$

Proof. Note that $\hat{u}_{i} \rightarrow \hat{u}$ in $L^{1}([a, b])$. Choose a subsequence $\left(\hat{u}_{i_{j}}\right)$ of $\left(\hat{u}_{i}\right)$ such that

$$
\lim _{j \rightarrow \infty} V\left(\hat{u}_{i_{j}},[a, b]\right)=\liminf _{i \rightarrow \infty} V\left(\hat{u}_{i},[a, b]\right)
$$

and then a subsequence of $\left(\hat{u}_{i_{j}}\right)$, denoted again by $\left(\hat{u}_{i_{j}}\right)$, such that $\hat{u}_{i_{j}} \rightarrow \hat{u}$ in $C$ where $m_{1}([a, b] \backslash C)=0$. From (2.2) we obtain

$$
\begin{aligned}
& V(\hat{u},[a, b])=V(\hat{u}, C) \leq \liminf _{j \rightarrow \infty} V\left(\hat{u}_{i_{j}}, C\right) \\
& =\liminf _{j \rightarrow \infty} V\left(\hat{u}_{i_{j}},[a, b]\right)=\liminf _{i \rightarrow \infty} V\left(\hat{u}_{i},[a, b]\right)
\end{aligned}
$$

as required.

$M_{1}$ - and $A M-$ modulus. A curve in $X$ is a continuous mapping $\gamma:[a, b] \rightarrow X$. We can always assume that a curve is non-constant and rectifiable and then we parametrize $\gamma$ by arc length, i.e. $\gamma:[0, l(\gamma)] \rightarrow X$ where $l(\gamma)$ is the length of $\gamma$. This is due to the fact that both the $M_{1}$ - and the $A M$-modulus of the family of all non-rectifiable curves in $X$ is zero. Note that it is essential that we consider curves which are defined on compact intervals. The $M_{1}$ - and $A M$-modulus can be defined on families of curves which are defined on open or half open intervals and which are only locally rectifiable. Then it turns out that there are families of non-rectifiable curves whose $M_{1}$ - and the $A M$-modulus are not zero.

Let $\Gamma$ be a family of curves in $X$. We first recall the concept of $M_{p}$-modulus, $p \geq 1$. A non-negative Borel function $\rho$ is $M_{p}-$ admissible for $\Gamma$ if

$$
\int_{\gamma} \rho d s \geq 1
$$

for every $\gamma \in \Gamma$. The $M_{p}-$ modulus of $\Gamma$ is defined as

$$
M_{p}(\Gamma)=\inf \int_{X} \rho^{p} d \nu
$$

where the infimum is taken over all admissible functions $\rho$. For the theory of $M_{p^{-}}$ modulus see [5,8. In this paper we only use the $M_{1}$-modulus.

A sequence of non-negative Borel functions $\rho_{i}, i=1,2, \ldots$, is $A M$-admissible for $\Gamma$ if

$$
\liminf _{i \rightarrow \infty} \int_{\gamma} \rho_{i} d s \geq 1
$$

for every $\gamma \in \Gamma$. The approximation modulus, $A M$-modulus for short, of $\Gamma$ is defined as

$$
A M(\Gamma)=\inf _{\left(\rho_{i}\right)}\left\{\liminf _{i \rightarrow \infty} \int_{X} \rho_{i} d \nu\right\}
$$

where the infimum is taken over all $A M$-admissibe sequences $\left(\rho_{i}\right)$ for $\Gamma$. 
We say that a property holds on $M_{p}$-almost every (a.e.) curve or on $A M$-a.e. curve if it holds for every curve except for a family $\Gamma$ with $M_{p}(\Gamma)=0$ or $A M(\Gamma)=0$, respectively. Since $A M(\Gamma) \leq M_{1}(\Gamma)$ for each curve family $\Gamma$ in $X$, the property which holds on $M_{1}$-a.e. curve holds on $A M$-a.e. curve as well. For the properties of the $A M$-modulus see 13 .

$B V_{A M}$ upper bounds. Let $\gamma$ be a curve in $X$ and $u$ a function such that $u \circ \gamma$ is a.e. defined in $[0, l(\gamma)]$. If $V(\widehat{u \circ \gamma},[0, l(\gamma)])<\infty$, we set $V(u, \gamma)=V(\widehat{u \circ \gamma},[0, l(\gamma)])$ and if there is no set $C \subset[0, l(\gamma)]$ such that $m_{1}([0, l(\gamma)] \backslash C)=0$ and $V(u \circ \gamma, C)<\infty$, then we set $V(u, \gamma)=\infty$.

Suppose that $u \in L^{1}(X)$. By [5, Lemma 1.43 and Proposition 1.37] the integral

$$
\int_{\gamma} u d s
$$

is well defined and finite on $M_{1}$-a.e. curve $\gamma$. In particular the function $u \circ \gamma$ is a.e. defined on $[0, l(\gamma)]$ and hence $V(u, \gamma)$ is defined on $M_{1}$ - and hence on $A M$-a.e. curve $\gamma$ in $X$.

A sequence $\left(g_{i}\right)$ of non-negative Borel functions is called a $B V_{A M}$ upper bound for $u \in L^{1}(X)$ if

$$
V(u, \gamma) \leq \liminf _{i \rightarrow \infty} \int_{\gamma} g_{i} d s
$$

on $A M$-a.e. curve $\gamma$ in $X$. Note that if $\left(g_{i}\right)$ is a $B V_{A M}$ upper bound for $u$, then every subsequence of $\left(g_{i}\right)$ is also a $B V_{A M}$ upper bound for $u$.

The next lemma gives alternative characterizations for (2.6).

Lemma 2.2. Let $u \in L^{1}(X)$ and $\left(g_{i}\right)$ a sequence of non-negative Borel functions in $X$. Then the following conditions are equivalent:

$\left(g_{i}\right)$ is a $B V_{A M}$ upper bound for $u$,

$$
\begin{aligned}
& V\left(u, \gamma \mid\left[t_{1}, t_{2}\right]\right) \leq \liminf _{i \rightarrow \infty} \int_{\gamma \mid\left[t_{1}, t_{2}\right]} g_{i} d s \text { for all } 0 \leq t_{1}<t_{2} \leq l(\gamma), \\
& \left|u\left(\gamma\left(t_{2}\right)\right)-u\left(\gamma\left(t_{1}\right)\right)\right| \leq \liminf _{i \rightarrow \infty} \int_{\gamma \mid\left[t_{1}, t_{2}\right]} g_{i} d s \text { for a.e. } t_{1}, t_{2} \in[0, l(\gamma)],
\end{aligned}
$$

where (2.8) and (2.9) are supposed to hold on AM-a.e. curve $\gamma$ in $X$.

Proof. Suppose that (2.7) holds. Since (2.6) holds on every curve $\gamma$, it holds on every subcurve of $\gamma$ as well and so (2.8) follows.

Clearly (2.8) implies (2.9) and it remains to show (2.9) $\Rightarrow$ (2.7). Suppose that (2.9) holds on a curve $\gamma$. Let $C \subset[0, l(\gamma]$ be a set of full measure and (2.9) holds for all $t_{1}, t_{2} \in C, t_{1}<t_{2}$ and let $a_{1}<a_{2}<\cdots<a_{n}$ be points in $C$. Then

$$
\begin{gathered}
\sum_{j=1}^{n-1} \mid u\left(\gamma\left(a_{j+1}\right)-u\left(\gamma\left(a_{j}\right)\right) \mid \leq \sum_{j=1}^{n-1} \liminf _{i \rightarrow \infty} \int_{\gamma \mid\left[a_{j}, a_{j+1}\right]} g_{i} d s\right. \\
\leq \liminf _{i \rightarrow \infty} \sum_{j=1}^{n-1} \int_{\gamma \mid\left[a_{j}, a_{j+1}\right]} g_{i} d s \leq \liminf _{i \rightarrow \infty} \int_{\gamma} g_{i} d s
\end{gathered}
$$

and this shows that $\left(g_{i}\right)$ is a $B V_{A M}$ upper bound for $u$. The lemma follows. 
Lemma 2.3. If $u_{i} \rightarrow u$ in $L^{1}(X)$, then the sequence $\left(u_{i}\right)$ has a subsequence $\left(u_{i_{j}}\right)$ such that

$$
V(u, \gamma) \leq \liminf _{j \rightarrow \infty} V\left(u_{i_{j}}, \gamma\right)
$$

for $A M-$-a.e. curve $\gamma$ in $X$.

Proof. By the Fuglede lemma [5, Lemma 2.1] the sequence $\left(u_{i}\right)$ has a subsequence $\left(u_{i_{j}}\right)$ such that

$$
\int_{\gamma}\left|u_{i_{j}}-u\right| d s \rightarrow 0
$$

as $j \rightarrow \infty$ for $M_{1}$-a.e., and thus for $A M$-a.e., curve $\gamma$ in $X$. The conclusion now follows from Lemma 2.1

If $\left(g_{i}\right)$ and $\left(h_{i}\right)$ are $B V_{A M}$ upper bounds of $u$ in $X$, then $\left(\max \left(g_{i}, h_{i}\right)\right)$ is clearly a $B V_{A M}$ upper bound for $u$ but, as easy examples show, $\left(\min \left(g_{i}, h_{i}\right)\right)$ is not. In the following lemma we consider the situation where the $B V_{A M}$ upper bounds are defined on different open sets.

Lemma 2.4. Let $\Omega_{j}, j=1,2$, be open sets in $X$ and $\left(g_{i}^{j}\right)$ a $B V_{A M}$ upper bound for a function $u \in L^{1}\left(\Omega_{1} \cup \Omega_{2}\right)$ in $\Omega_{j}, j=1,2$. Then the sequence $\left(g_{i}\right)$,

$$
g_{i}(x)=\left\{\begin{array}{l}
g_{i}^{1}(x), x \in \Omega_{1} \backslash \Omega_{2}, \\
\max \left(g_{i}^{1}(x), g_{i}^{2}(x)\right), x \in \Omega_{1} \cap \Omega_{2}, \\
g_{i}^{2}(x), x \in \Omega_{2} \backslash \Omega_{1},
\end{array}\right.
$$

is a $B V_{A M}$ upper bound for $u$ in $\Omega_{1} \cup \Omega_{2}$.

Proof. Note that $\left(g_{i}\right)$ is a $B V_{A M}$ upper bound for $u$ both in $\Omega_{1}$ and $\Omega_{2}$ and hence

$$
V\left(u, \gamma^{\prime}\right) \leq \liminf _{i \rightarrow \infty} \int_{\gamma^{\prime}} g_{i} d s
$$

whenever $\gamma^{\prime}$ is a subcurve of $\gamma$ with $\gamma^{\prime} \subset \Omega_{j}, j=1$ or 2 .

Fix a curve $\gamma$ in $\Omega_{1} \cup \Omega_{2}$. Since $\gamma([0, l(\gamma)])$ is compact in $\Omega_{1} \cup \Omega_{2}$, there is $\delta>0$ such that $\gamma^{\prime}$ lies in $\Omega_{1}$ or in $\Omega_{2}$ whenever $\gamma^{\prime}$ is a subcurve of $\gamma$ with $l\left(\gamma^{\prime}\right)<\delta$. By (2.10) and Lemma 2.2 there is a set $C \subset[0, l(\gamma)]$ with $m_{1}([0, l(\gamma)] \backslash C)=0$ and

$$
\left|u\left(\gamma\left(t_{2}\right)\right)-u\left(\gamma\left(t_{1}\right)\right)\right| \leq \liminf _{i \rightarrow \infty} \int_{\gamma \mid\left[t_{1}, t_{2}\right]} g_{i} d s
$$

whenever $t_{1}, t_{2} \in C, 0<t_{2}-t_{1}<\delta$. Let $0 \leq t_{0}<t_{1}<\cdots<t_{n} \leq l(\gamma), t_{k} \in C$, $k=0,1, \ldots, n$. Adding a finite number of points from $C$ to the sequence $\left(t_{k}\right)$ we can assume that $t_{k}-t_{k-1}<\delta$ for each $k$. Now (2.11) yields

$$
\begin{gathered}
\sum_{k=1}^{n}\left|u\left(\gamma\left(t_{k}\right)\right)-u\left(\gamma\left(t_{k-1}\right)\right)\right| \leq \sum_{k=1}^{n} \liminf _{i \rightarrow \infty} \int_{\gamma \mid\left[t_{k}, t_{k-1}\right]} g_{i} d s \\
\leq \liminf _{i \rightarrow \infty} \sum_{k=1}^{n} \int_{\gamma \mid\left[t_{k}, t_{k-1}\right]} g_{i} d s \leq \liminf _{i \rightarrow \infty} \int_{\gamma} g_{i} d s
\end{gathered}
$$

and the lemma follows. 


\section{The SPACE $B V_{A M}(X)$}

The space, abreviated as $B V_{A M}(X)$, of the functions of bounded variation on $A M$-a.e. curve in $X$ consists of all functions $u \in L^{1}(X)$ which have a $B V_{A M}$ upper bound $\left(g_{i}\right)$ such that

$$
\liminf _{i \rightarrow \infty} \int_{X} g_{i} d \nu<\infty
$$

We call

where

$$
\|u\|_{B V(X)}=\|u\|_{L^{1}(X)}+\left\|D_{B V} u\right\|(X)
$$

$$
\left\|D_{B V} u\right\|(X)=\inf \left\{\liminf _{i \rightarrow \infty} \int_{X} g_{i} d \nu:\left(g_{i}\right) \text { is a } B V_{A M} \text { upper bound for } u\right\}
$$

the $B V_{A M}$ norm of $u$ in $X$. The essential implication of (3.1) is the following; see [13]. For completeness we give a proof.

Lemma 3.1. Suppose that $u \in L^{1}(X)$ has a $B V_{A M}$ upper bound $\left(g_{i}\right)$ in $X$ as in (3.1). Then

$$
V(u, \gamma) \leq \liminf _{i \rightarrow \infty} \int_{\gamma} g_{i} d s<\infty
$$

for $A M-$-a.e. curve $\gamma$ in $X$.

Proof. For each $k=1,2, \ldots$ let $\Gamma_{k}$ be the family of all curves $\gamma$ in $X$ such that

$$
\liminf _{i \rightarrow \infty} \int_{\gamma} g_{i} d s \geq k
$$

Then the sequence $\left(g_{i} / k\right)$ is $A M$-admissible for $\Gamma_{k}$ and hence

$$
A M\left(\Gamma_{k}\right) \leq \liminf _{i \rightarrow \infty} \int_{X} \frac{g_{i}}{k} d \nu=\frac{M}{k}
$$

where

$$
M=\liminf _{i \rightarrow \infty} \int_{X} g_{i} d \nu
$$

Since

$$
\Gamma_{\infty}=\left\{\gamma: \liminf _{i \rightarrow \infty} \int_{\gamma} g_{i} d s=\infty\right\}
$$

satisfies $\Gamma_{\infty} \subset \Gamma_{k}$ for each $k$, it follows that $A M\left(\Gamma_{\infty}\right)=0$ and thus (3.2) holds as required.

The following property of a function $u \in B V_{A M}(X)$ is often useful. For each $\varepsilon>0$ there is a $B V_{A M}$ upper bound $\left(g_{i}\right)$ for $u$ in $X$ such that

$$
\int_{X} g_{i} d \nu \leq\left\|D_{B V} u\right\|(X)+\varepsilon
$$

for all $i$ and (3.2) holds for $A M$-a.e. curve $\gamma$ in $X$.

Remark 3.2. A locally Lipschitz function $u \in L^{1}(X)$ with an upper gradient also in $L^{1}(X)$ and a function $u$ in the Newtonian space $N^{1,1}(X)$ is in the space $B V_{A M}(X)$ since for such a function $u$ the sequence $\left(g_{i}\right)$, where $g_{i}=g$ and $g$ is an upper gradient or a 1-weak upper gradient of $u$, respectively, is a $B V_{A M}$ upper bound for $u$. For the concept of an upper gradient and a 1-weak upper gradient see [4, 5, 6, 15]. 
Lemma 3.3. The space $B V_{A M}(X)$ is a linear subspace of $L^{1}(X)$ and $\|u\|_{B V(X)}$ is a norm in $B V_{A M}(X)$.

Proof. If $u \in B V_{A M}(X)$ and $\lambda \in \mathbf{R}$, then obviously $\lambda u \in B V_{A M}(X)$ and $\|\lambda u\|_{B V(X)}=|\lambda|\|u\|_{B V(X)}$. If $u=0$ in $L^{1}(X)$, then $u \circ \gamma=0$ a.e. in $[0, l(\gamma)]$ on $M_{1}$-a.e. curve $\gamma$ in $X$; see Section 2 . Hence $\left(g_{i}\right), g_{i}=0, i=1,2, \ldots$, is a $B V_{A M}$ upper bound for $u$ and thus $\|u\|_{B V(X)}=0$.

It remains to show that $u+v \in B V_{A M}(X)$ provided that $u, v \in B V_{A M}(X)$ and that the triangle inequality holds. Let $u, v \in B V_{A M}(X)$ and let $\left(g_{i}\right)$ and $\left(h_{i}\right)$ be $B V_{A M}$ upper bounds for $u$ and $v$, respectively. Now $\left(g_{i}+h_{i}\right)$ is a $B V_{A M}$ upper bound for $u+v$. Indeed, for $A M$-a.e. curve $\gamma$ in $X$

$$
\begin{gathered}
V(u+v, \gamma) \leq V(u, \gamma)+V(v, \gamma) \\
\leq \liminf _{i \rightarrow \infty} \int_{\gamma} g_{i} d s+\liminf _{i \rightarrow \infty} \int_{\gamma} h_{i} d s \leq \liminf _{i \rightarrow \infty} \int_{\gamma}\left(g_{i}+h_{i}\right) d s
\end{gathered}
$$

and thus $u+v \in B V_{A M}(X)$. For the triangle inequality note that the $B V_{A M}$ upper bounds $\left(g_{i}\right)$ and $\left(h_{i}\right)$ can be chosen so that for $\varepsilon>0$

$$
\left\|D_{B V} u\right\|(X) \geq \int_{X} g_{i} d \nu-\varepsilon,\left\|D_{B V} v\right\|(X) \geq \int_{X} h_{i} d \nu-\varepsilon
$$

for every $i$. Since $\left(g_{i}+h_{i}\right)$ is a $B V_{A M}$ upper bound for $u+v$, the triangle inequality easily follows from the above property of $\left(g_{i}\right)$ and $\left(h_{i}\right)$.

Theorem 3.4. The space $B V_{A M}(X)$ with the norm $\|u\|_{B V(X)}$ is a Banach space.

Proof. It remains to show the Cauchy property. Let $u_{i}, i=1,2, \ldots$, be a Cauchy sequence in $B V_{A M}(X)$. Since $u_{i} \rightarrow u$ in $L^{1}(X)$, it suffices to show that a subsequence, denoted again by $\left(u_{i}\right)$, satisfies $\left\|D_{B V}\left(u_{i}-u\right)\right\|(X) \rightarrow 0$ as $i \rightarrow \infty$ and $u \in B V_{A M}(X)$. By the Fuglede lemma (see [5, Lemma 2.1]), we may assume that

$$
\int_{\gamma}\left|u_{i}-u\right| d s \rightarrow 0
$$

as $i \rightarrow \infty$ for $M_{1}$-a.e. curve $\gamma$ in $X$ and

$$
\left\|D_{B V}\left(u_{i+1}-u_{i}\right)\right\|(X)<2^{-i}
$$

for each $i$. From (3.5) it follows that for every $i$ there is a $B V_{A M}$ upper bound $\left(g_{j}^{i}\right)$ for $u_{i+1}-u_{i}$ such that

$$
\left\|D_{B V}\left(u_{i+1}-u_{i}\right)\right\|(X) \leq \int_{X} g_{j}^{i} d \nu<2^{-i}
$$

for all $j=1,2, \ldots$.

For $k=1,2, \ldots$ consider the sequence

$$
\tilde{g}_{j}^{k}=\sum_{l=k}^{\infty} g_{j}^{l}, j=1,2, \ldots
$$


Since $\tilde{g}_{j}^{k} \geq g_{j}^{i}$ for each $i \geq k,\left(\tilde{g}_{j}^{k}\right)$ is a $B V_{A M}$ upper bound for $u_{i+1}-u_{i}, i \geq k$. Now $\tilde{g}_{j}^{k}$ is a $B V_{A M}$ upper bound for $u_{i+1}-u_{k}, i \geq k$ as well, because

$$
\begin{aligned}
V\left(u_{i+1}-u_{k}, \gamma\right) & \leq \sum_{l=k}^{i} V\left(u_{l+1}-u_{l}, \gamma\right) \leq \sum_{l=k}^{i} \liminf _{j \rightarrow \infty} \int_{\gamma} g_{j}^{l} d s \\
& \leq \liminf _{j \rightarrow \infty} \sum_{l=k}^{i} \int_{\gamma} g_{j}^{l} d s \leq \liminf _{j \rightarrow \infty} \int_{\gamma} \tilde{g}_{j}^{k} d s,
\end{aligned}
$$

and this holds for $A M$-a.e. curve $\gamma$ by the subadditivity of the $A M$-modulus.

Next we show that $\left(\tilde{g}_{j}^{k}\right)$ is a $B V_{A M}$ upper bound for $u-u_{k}$. For this fix a curve $\gamma$ such that (3.4) holds and that

$$
V\left(u_{i+1}-u_{k}, \gamma\right) \leq \liminf _{j \rightarrow \infty} \int_{\gamma} \tilde{g}_{j}^{k} d s
$$

for every $i \geq k$. Note that $A M$-a.e. curve $\gamma$ satisfies these conditions. By Lemma 2.1

$$
\begin{aligned}
& V\left(u-u_{k}, \gamma\right) \leq \liminf _{i \rightarrow \infty} V\left(u_{i+1}-u_{k}, \gamma\right) \\
\leq & \liminf _{i \rightarrow \infty} \liminf _{j \rightarrow \infty} \int_{\gamma} \tilde{g}_{j}^{k} d s=\liminf _{j \rightarrow \infty} \int_{\gamma} \tilde{g}_{j}^{k} d s
\end{aligned}
$$

and hence $\left(\tilde{g}_{j}^{k}\right)$ is a $B V_{A M}$ upper bound for $u-u_{k}$. From (3.6) it follows that

$$
\begin{gathered}
\left\|D_{B V}\left(u-u_{k}\right)\right\|(X) \leq \liminf _{j \rightarrow \infty} \int_{X} \tilde{g}_{j}^{k} d \nu \\
=\liminf _{j \rightarrow \infty} \sum_{l=k}^{\infty} \int_{X} g_{j}^{l} d \nu \leq \liminf _{j \rightarrow \infty} \sum_{l=k}^{\infty} \frac{1}{2^{l}}=\frac{2}{2^{k}} .
\end{gathered}
$$

Thus $u-u_{k} \in B V_{A M}(X)$ and so $u \in B V_{A M}(X)$. Inequality (3.7) also shows that $\left\|D_{B V}\left(u-u_{k}\right)\right\|(X) \rightarrow 0$ as $k \rightarrow \infty$. This completes the proof.

In 14. Miranda considered functions $u \in L^{1}(X)$ which can be approximated in $L^{1}(X)$ by locally Lipschitz functions whose upper gradients belong to $L^{1}(X)$. Set $(3.8)$

$$
\left\|D_{L} u\right\|(X)=\inf \left\{\liminf _{i \rightarrow \infty} \int_{X} g_{i} d \nu: u_{i} \rightarrow u \text { in } L^{1}(X), u_{i} \text { loc. Lipschitz }\right\}<\infty
$$

where $g_{i}$ is an upper gradient of $u_{i}$ and the first infimum is taken over all sequences of locally Lipschitz functions $\left(u_{i}\right)$ in $X$ such that $u_{i} \rightarrow u$ in $L^{1}(X)$ and all upper gradients $g_{i}$ of $u_{i}$. The space of all functions such that

$$
\|u\|_{L}(X)=\|u\|_{L^{1}(X)}+\left\|D_{L} u\right\|(X)<\infty
$$

is a Banach space and the space is a generalization of the ordinary space of functions of bounded variation in $\mathbf{R}^{n}$; see 7 .

The functions in the above space are also of bounded variation on $A M$-a.e. curve in $X$ (see [13, Theorem 5.4]) in the sense that $V(u, \gamma)<\infty$ on $A M$-a.e. curve in $X$. More precisely, if $\left(u_{i}\right)$ is a sequence of locally Lipschitz functions with upper gradients $g_{i}$ in $X$ and $u_{i} \rightarrow u$ in $L^{1}(X)$, then there is a subsequence $\left(u_{i_{j}}\right)$ such that

$$
\int_{\gamma}\left|u_{i_{j}}-u\right| d s \rightarrow 0
$$


on $M_{1}$-a.e. curve $\gamma$ in $X$ and (see Lemma 2.3) that

$$
V(u, \gamma) \leq \liminf _{j \rightarrow \infty} V\left(u_{i_{j}}, \gamma\right) \leq \liminf _{j \rightarrow \infty} \int_{\gamma} g_{i_{j}} d s
$$

where $g_{i_{j}}$ is an upper gradient of $u_{i_{j}}$. Thus the sequence $\left(g_{i_{j}}\right)$ is a $B V_{A M}$ upper bound for $u$. Hence

$$
\left\|D_{B V} u\right\|(X) \leq\left\|D_{L} u\right\|(X)
$$

holds for every function $u \in L^{1}(X)$.

Remark 3.5. It would be interesting to know if the inequality

$$
\left\|D_{B V} u\right\|(\Omega)<\left\|D_{L} u\right\|(\Omega)
$$

could hold in some open set $\Omega \subset X$ in the case where functions in $L^{1}(X)$ can be approximated by locally Lipschitz functions.

\section{The measure associated with $\left\|D_{B V} u\right\|$}

We show that, under some additional assumptions on $X$, for each $u \in B V_{A M}(X)$ there is a Borel measure $\mu=\mu_{u}$ defined initially on open subsets $\Omega$ of $X$ as $\mu(\Omega)=$ $\left\|D_{B V} u\right\|(\Omega)$ and then extended to arbitrary sets $A \subset X$ as

$$
\mu(A)=\inf \{\mu(\Omega): A \subset \Omega, \Omega \text { open }\} .
$$

This procedure (see [9]) requires that the space $(X, d)$ is separable and locally compact. These assumptions imply that the metric space $X$ is $\sigma$-compact and hence open sets $\Omega$ in $X$ can be exhausted by compact sets and, in particular, by open sets with compact closure in $\Omega$. If the measure $\nu$ is doubling and the space $X$ is complete, then $X$ is proper, i.e. closed and bounded sets are compact, so that these assumptions imply separability and local compactness. For the discussion on these conditions see [5]. Miranda [14] also used this construction for the corresponding measure based on $\left\|D_{L} u\right\|(\Omega)$.

In this section we assume that $X$ is separable and locally compact. The measure $\nu$ in $X$ is supposed to satisfy (2.1).

Theorem 4.1. If $u \in B V_{A M}(X)$, then the set function $\mu(\Omega)=\left\|D_{B V} u\right\|(\Omega), \Omega$ open in $X$, defines a Borel measure in $X$.

Proof. By [9, Theorem 5.1] it suffices to show that for open sets $\Omega, \Omega_{1}$ and $\Omega_{2}$ in $X$ it holds that

$$
\begin{gathered}
\mu\left(\Omega_{1}\right) \leq \mu\left(\Omega_{2}\right), \text { if } \Omega_{1} \subset \Omega_{2}, \\
\mu\left(\Omega_{1} \cup \Omega_{2}\right)=\mu\left(\Omega_{1}\right)+\mu\left(\Omega_{2}\right), \text { if } \Omega_{1} \cap \Omega_{2}=\emptyset, \\
\mu\left(\Omega_{1} \cup \Omega_{2}\right) \leq \mu\left(\Omega_{1}\right)+\mu\left(\Omega_{2}\right), \\
\mu(\Omega)=\sup \left\{\mu\left(\Omega_{1}\right): \Omega_{1} \subset \subset \Omega\right\} .
\end{gathered}
$$

Here $A \subset \subset B$ means that the closure of $A$ is a compact subset of $B$.

Now (4.1) is obvious and (4.2) follows from the fact that each curve in $\Omega_{1} \cup \Omega_{2}$ lies either in $\Omega_{1}$ or in $\Omega_{2}$. It remains to verify (4.3) and (4.4). 
To prove (4.3) let $\varepsilon>0$ and choose $B V_{A M}$ upper bounds $\left(g_{i}^{1}\right)$ and $\left(g_{i}^{2}\right)$ for $u$ in $\Omega_{1}$ and $\Omega_{2}$, respectively, so that

$$
\left\|D_{B V} u\right\|\left(\Omega_{j}\right) \geq \int_{\Omega_{j}} g_{i}^{j} d \nu-\varepsilon
$$

for all $i$ and $j=1,2$. Define $g_{i}=g_{i}^{1}$ in $\Omega_{1} \backslash \Omega_{2}, g_{i}=g_{i}^{2}$ in $\Omega_{2} \backslash \Omega_{1}$ and $g_{i}=$ $\max \left(g_{i}^{1}, g_{i}^{2}\right)$ in $\Omega_{1} \cap \Omega_{2}$. Then by Lemma 2.4, $\left(g_{i}\right)$ is a $B V_{A M}$ upper bound for $u$ in $\Omega_{1} \cup \Omega_{2}$ and we obtain from (4.5)

$$
\begin{gathered}
\left\|D_{B V} u\right\|\left(\Omega_{1} \cup \Omega_{2}\right) \leq \liminf _{i \rightarrow \infty} \int_{\Omega_{1} \cup \Omega_{2}} g_{i} d \nu \\
\leq \liminf _{i \rightarrow \infty}\left(\int_{\Omega_{1} \backslash \Omega_{2}} g_{i}^{1} d \nu+\int_{\Omega_{2} \backslash \Omega_{1}} g_{i}^{2} d \nu+\int_{\Omega_{1} \cap \Omega_{2}}\left(g_{i}^{1}+g_{i}^{2}\right) d \nu\right) \\
\leq\left\|D_{B V} u\right\|\left(\Omega_{1}\right)+\left\|D_{B V} u\right\|\left(\Omega_{2}\right)+2 \varepsilon .
\end{gathered}
$$

Letting $\varepsilon \rightarrow 0$ we complete the proof for (4.3).

To prove (4.4) write

$$
\alpha=\sup \left\{\mu\left(\Omega_{1}\right): \Omega_{1} \subset \subset \Omega\right\} .
$$

We may assume that $\alpha<\infty$. Let $\varepsilon>0$ and pick an open set $U_{0} \subset \subset \Omega$ such that

$$
\left\|D_{B V} u\right\|\left(U_{0}\right)>\alpha-\varepsilon \text {. }
$$

Next pick open subsets $U_{i}$ and $V_{i}, i=1,2, \ldots$, such that

$$
U_{0} \subset \subset V_{1} \subset \subset U_{1} \subset \subset V_{2} \subset \subset U_{2} \subset \subset \cdots \subset \subset \Omega
$$

and $\bigcup_{i=1}^{\infty} U_{i}=\Omega$. Note that by (4.6) and (4.1) we have

$$
\left\|D_{B V} u\right\|\left(U_{1}\right)>\alpha-\varepsilon \text {. }
$$

The open sets $U_{i} \backslash \bar{U}_{i-1}, i \geq 2$, are disjoint and lie in $\Omega \backslash \bar{U}_{1}$. Hence by (4.2) for each $j \geq 2$,

$$
\left\|D_{B V} u\right\|\left(\bigcup_{i=2}^{j}\left(U_{i} \backslash \bar{U}_{i-1}\right)\right)=\sum_{i=2}^{j}\left\|D_{B V} u\right\|\left(U_{i} \backslash \bar{U}_{i-1}\right)
$$

and we obtain

$$
\left.\left\|D_{B V} u\right\|\left(U_{1}\right)+\left\|D_{B V} u\right\|\left(\bigcup_{i=2}^{j}\left(U_{i} \backslash \bar{U}_{i-1}\right)\right)\right) \leq \alpha
$$

and thus (4.7) yields

$$
\sum_{i=2}^{j}\left\|D_{B V} u\right\|\left(U_{i} \backslash \bar{U}_{i-1}\right)<\varepsilon
$$

for every $j \geq 2$. Similarly we obtain

$$
\sum_{i=1}^{j}\left\|D_{B V} u\right\|\left(V_{i+1} \backslash \bar{V}_{i}\right)<\varepsilon
$$

for every $j \geq 1$.

Choose a $B V_{A M}$ upper bound $\left(g_{k}^{1}\right)$ for $u$ in $U_{1}$ such that

$$
\int_{U_{1}} g_{k}^{1} d \nu<\alpha+\varepsilon
$$


and for every $i=2,3, \ldots$, a $B V_{A M}$ upper bound $\left(g_{k}^{i}\right)$ for $u$ in $U_{i} \backslash \bar{U}_{i-1}$ such that

$$
\int_{U_{i} \backslash \bar{U}_{i-1}} g_{k}^{i} d \nu<\left\|D_{B V} u\right\|\left(U_{i} \backslash \bar{U}_{i-1}\right)+\frac{\varepsilon}{2^{i}}
$$

for all $k=1,2, \ldots$. Similarly for $i=1,2, \ldots$ we choose $B V_{A M}$ upper bounds $\left(h_{k}^{i}\right)$ for $u$ in $V_{i+1} \backslash \bar{V}_{i}$ such that

$$
\int_{V_{i+1} \backslash \bar{V}_{i}} h_{k}^{i} d \nu<\left\|D_{B V} u\right\|\left(V_{i+1} \backslash \bar{V}_{i}\right)+\frac{\varepsilon}{2^{i}}
$$

for each $k$. Since (4.9) and (4.10) hold for each $j$, (4.12) and (4.13) yield

$$
\sum_{i=2}^{\infty} \int_{U_{i} \backslash \bar{U}_{i-1}} g_{k}^{i} d \nu \leq \sum_{i=2}^{\infty}\left\|D_{B V} u\right\|\left(U_{i} \backslash \bar{U}_{i-1}\right)+\varepsilon \leq 2 \varepsilon
$$

and

$$
\sum_{i=1}^{\infty} \int_{V_{i+1} \backslash \bar{V}_{i}} h_{k}^{i} d \nu \leq \sum_{i=1}^{\infty}\left\|D_{B V} u\right\|\left(V_{i+1} \backslash \bar{V}_{i}\right)+\varepsilon \leq 2 \varepsilon .
$$

Extend each of the functions $g_{k}^{i}$ and $h_{k}^{i}$ as zero outside their domains of definition and let

$$
\tilde{g}_{k}=\sum_{i=1}^{\infty}\left(g_{k}^{i}+h_{k}^{i}\right), k=1,2, \ldots .
$$

Now $\left(\tilde{g}_{k}\right)$ is a $B V_{A M}$ upper bound for $u$ in $\Omega$. To see this note that for a fixed $k$ the sum $\sum_{i=1}^{\infty}\left(g_{k}^{i}(x)+h_{k}^{i}(x)\right)$ contains at most two non-zero terms for each $x \in \Omega$ and that

$$
\Omega=U_{1} \cup \bigcup_{i=2}^{\infty}\left(U_{i} \backslash \bar{U}_{i-1}\right) \cup \bigcup_{i=1}^{\infty}\left(V_{i+1} \backslash \bar{V}_{i}\right) .
$$

Fix a curve $\gamma$ in $\Omega$. Since $\gamma([0, l(\gamma)])$ is a compact subset of $\Omega$, there is $j$ such that

$$
\gamma([0, l(\gamma)]) \subset U_{1} \cup \bigcup_{i=2}^{j}\left(U_{i} \backslash \bar{U}_{i-1}\right) \cup \bigcup_{i=1}^{j}\left(V_{i+1} \backslash \bar{V}_{i}\right)=V_{j+1} .
$$

On the other hand,

$$
\tilde{g}_{k}(x) \geq g_{k}^{1}(x)+\max _{2 \leq i \leq j} g_{k}^{i}(x)+\max _{1 \leq i \leq j} h_{k}^{i}(x)=w_{k}^{j}(x)
$$

for each $x \in V_{j+1}$ and every $k$ and, since by Lemma 2.4 $\left(w_{k}^{j}\right)$ is a $B V_{A M}$ upper bound for $u$ in $V_{j+1},\left(\tilde{g}_{k}\right)$ is a $B V_{A M}$ upper bound for $u$ in $\Omega$ as required. Consequently,

$$
\begin{gathered}
\left\|D_{B V} u\right\|(\Omega) \leq \liminf _{k \rightarrow \infty} \int_{\Omega} \tilde{g}_{k} d \nu \\
\leq \liminf _{k \rightarrow \infty}\left(\int_{U_{1}} g_{k}^{1} d \nu+\sum_{i=2}^{\infty} \int_{U_{i} \backslash \bar{U}_{i-1}} g_{k}^{i} d \nu+\sum_{i=1}^{\infty} \int_{V_{i} \backslash \bar{V}_{i-1}} h_{k}^{i} d \nu\right) \\
\leq \liminf _{k \rightarrow \infty}\left(\int_{U_{1}} g_{k}^{1} d \nu+\sum_{i=2}^{\infty}\left\|D_{B V} u\right\|\left(V_{i} \backslash \bar{V}_{i-1}\right)+\sum_{i=1}^{\infty}\left\|D_{B V} u\right\|\left(V_{i} \backslash \bar{V}_{i-1}\right)\right) \\
\leq \alpha+\varepsilon+2 \varepsilon+2 \varepsilon=\alpha+5 \varepsilon
\end{gathered}
$$

where we have used (4.11), (4.14) and (4.15). Since $\varepsilon>0$ is arbitrary, $\left\|D_{B V} u\right\|(\Omega)$ $\leq \alpha$ and the proof for (4.4) follows and completes the proof for the theorem. 


\section{The measure $\left\|D_{B V} u\right\|$ IN $\mathbf{R}^{n}$}

We show that for $X=\mathbf{R}^{n}$ the space $B V_{A M}(X)$ is the ordinary space of $\mathrm{BV}$ functions. The simplest domain $\Omega$ in $\mathbf{R}^{n}$ from the modulus and the Fubini theorem point of view is the Cartesian product $\Omega=\left(a_{1}, b_{1}\right) \times\left(a_{2}, b_{2}\right) \times \cdots \times\left(a_{n}, b_{n}\right)$. We let $\Omega^{\prime}=\left(a_{1}, b_{1}\right) \times\left(a_{2}, b_{2}\right) \times \cdots \times\left(a_{n-1}, b_{n-1}\right)$ and $(a, b)=\left(a_{n}, b_{n}\right)$ so that $\Omega=\Omega^{\prime} \times(a, b)$. There is nothing particular in the choice of the last coordinate.

The classical space $B V(\Omega)$ of BV functions in $\Omega$ consists of all functions $u \in$ $L^{1}(\Omega)$ such that

$$
\|D u\|(\Omega)=\sup \left\{\int_{\Omega} u \nabla \cdot \varphi d m_{n}: \varphi \in C_{0}^{1}\left(\Omega, \mathbf{R}^{n}\right),|\varphi| \leq 1\right\}<\infty ;
$$

see [7, Section 5.1]. Note that Lipschitz functions with compact support can be considered as well as functions $\varphi$ in $C_{0}^{1}\left(\Omega, \mathbf{R}^{n}\right)$. Now $\|D u\|(U)$ and $U$ an open subset of $\Omega$, defines a Borel measure in $\Omega$.

In the following we let $X=\Omega$ and $\nu=m_{n}$ be the Lebesgue measure and consider the behavior of $V(u, \gamma)$ on line segments and the measure $\mu=\mu_{u}=\left\|D_{B V} u\right\|$, $u \in L^{1}(\Omega)$, defined in Section 4 .

For $y \in \Omega^{\prime}$ let $\gamma_{y}(t)=y+(a+t) e_{n}, t \in[0, b-a]$, where $e_{n}=(0,0, \ldots, 1)$. Then $\gamma_{y}$ is a straight line segment parallel to the $x_{n}$-axis. Note that $\gamma_{y}$ is a curve in $\Omega^{\prime} \times[a, b]$ but not in $\Omega$. However, for $u \in L^{1}(\Omega), V\left(u, \gamma_{y}\right)$ is well defined; see Section 2. The following lemma shows that the behavior of $u$ is similar on $\gamma_{y}$ as on curves in $\Omega$.

Lemma 5.1. Let $u \in L^{1}(\Omega), U$ an open subset of $\Omega^{\prime}$ and $\left(g_{i}\right)$ a $B V_{A M}$ upper bound for $u$ in $U \times(a, b)$ with

$$
\liminf _{i \rightarrow \infty} \int_{U \times(a, b)} g_{i} d m_{n}<\infty .
$$

Then for $m_{n-1}-$ a.e. $y \in U$ we have

$$
V\left(u, \gamma_{y}\right) \leq \liminf _{i \rightarrow \infty} \int_{\gamma_{y}} g_{i} d s<\infty .
$$

Proof. For $y \in U$ and $j>(b-a) / 2$ let $\gamma_{y}^{j}(t)=y+(a+1 / j+t) e_{n}, t \in[0, b-a-2 / j]$. Then each curve in the family $\Gamma^{j}=\left\{\gamma_{y}^{j}\right\}$ lies in $U \times(a, b)$ and hence for $A M$-a.e. $\gamma_{y}^{j} \in \Gamma^{j}$ we have

$$
V\left(u, \gamma_{y}^{j}\right) \leq \liminf _{i \rightarrow \infty} \int_{\gamma_{y}^{j}} g_{i} d s<\infty
$$

Now (5.2) holds for $m_{n-1}$-a.e. $y \in U$ because $A M\left(\Gamma^{j}\right)=m_{n-1}(U)$; see [13, Example 3.9].

The Fatou lemma yields

$$
\int_{U}\left(\liminf _{i \rightarrow \infty} \int_{\gamma_{y}} g_{i} d s\right) d m_{n-1}(y) \leq \liminf _{i \rightarrow \infty} \int_{U \times(a, b)} g_{i} d m_{n}<\infty
$$

and hence

$$
V\left(u, \gamma_{y}^{j}\right) \leq \liminf _{i \rightarrow \infty} \int_{\gamma_{y}} g_{i} d s<\infty
$$


for $m_{n-1}$-a.e. $y \in U$ for each $j$. From this it follows that

$$
V\left(u, \gamma_{y}\right)=\lim _{j \rightarrow \infty} V\left(u, \gamma_{y}^{j}\right) \leq \liminf _{i \rightarrow \infty} \int_{\gamma_{y}} g_{i} d s<\infty
$$

as required.

Lemma 5.2. Suppose that $u \in B V_{A M}(\Omega)$. Then the function $y \mapsto V\left(u, \gamma_{y}\right)$ belongs to $L^{1}\left(\Omega^{\prime}\right)$ with respect to the $m_{n-1}$ measure and

$$
\int_{\Omega^{\prime}} V\left(u, \gamma_{y}\right) d m_{n-1} \leq\left\|D_{B V} u\right\|(\Omega) .
$$

Proof. We first show that the function $V\left(u, \gamma_{y}\right)=V\left(\widehat{u \circ \gamma_{y}},[0, b-a]\right)$ is measurable in $\Omega^{\prime}$. To see this note that $\widehat{u \circ \gamma_{y}}(t)=u(y, \gamma(t))$ for a.e. $(y, t) \in \Omega^{\prime} \times[0, b-a]$ and thus $\widehat{u \circ \gamma_{y}}$ is a measurable function in $\Omega^{\prime} \times[0, b-a]$ and, moreover, an $L^{1}\left(\Omega^{\prime} \times[0, b-a]\right)$-function. Pick a set $C \subset[0, b-a]$ such that $m_{1}([0, b-a] \backslash C)=0$ and for every $t \in C$ the function $y \mapsto \widehat{u \circ \gamma_{y}}(t)$ is $m_{n-1}-$ measurable in $\Omega^{\prime}$. This is possible by the Fubini theorem. Thus if the points $t_{1}<t_{2}<\cdots<t_{m}$ lie in $C$, then the function

$$
y \mapsto \sum_{i=2}^{m}\left|\widehat{u \circ \gamma_{y}}\left(t_{i}\right)-\widehat{u \circ \gamma_{y}}\left(t_{i-1}\right)\right|
$$

is measurable in $\Omega^{\prime}$. For each $j=2,3, \ldots$ choose a finite increasing sequence $\left(t_{i}^{j}\right)$, $i=1,2, \ldots, j$ of points in $C$ such that $\max _{i}\left|t_{i}^{j}-t_{i-1}^{j}\right| \rightarrow 0$ as $j \rightarrow \infty$. Now the continuity properties of the function $\widehat{u \circ \gamma_{y}}$ imply that

$$
V\left(\widehat{u \circ \gamma_{y}}, C\right)=\limsup _{j \rightarrow \infty} \sum_{i=2}^{j}\left|\widehat{u \circ \gamma_{y}}\left(t_{i}\right)-\widehat{u \circ \gamma_{y}}\left(t_{i-1}\right)\right|
$$

and thus $y \mapsto V\left(u, \gamma_{y}\right)$ is measurable in $\Omega^{\prime}$.

Let $\varepsilon>0$ and choose a $B V_{A M}$ upper bound $\left(g_{i}\right)$ for $u$ in $\Omega$ with

$$
\liminf _{i \rightarrow \infty} \int_{\Omega} g_{i} d m_{n} \leq\left\|D_{B V} u\right\|(\Omega)+\varepsilon .
$$

By Lemma 5.1 and the Fatou lemma we have

$$
\begin{gathered}
\int_{\Omega^{\prime}} V\left(u, \gamma_{y}\right) d m_{n-1} \leq \int_{\Omega^{\prime}}\left(\liminf _{i \rightarrow \infty} \int_{\gamma_{y}} g_{i} d s\right) d m_{n-1} \\
\leq \liminf _{i \rightarrow \infty} \int_{\Omega} g_{i} d m_{n} \leq\left\|D_{B V} u\right\|(\Omega)+\varepsilon,
\end{gathered}
$$

and letting $\varepsilon \rightarrow 0$ we obtain (5.3).

The measure $\left\|D_{B V} u\right\|$ defines a Borel measure $\hat{\mu}$ in $\Omega^{\prime}$ by the rule $\hat{\mu}(A)=$ $\left\|D_{B V} u\right\|(A \times(a, b))$ for each Borel set $A \subset \Omega^{\prime}$. The next theorem clarifies the roles of $V\left(u, \gamma_{y}\right)$ and $\hat{\mu}$.

Lemma 5.3. Let $u \in B V_{A M}(\Omega)$. Then for $m_{n-1}$-a.e. $y \in \Omega^{\prime}$

$$
V\left(u, \gamma_{y}\right) \leq d \hat{\mu}(y)
$$

where $d \hat{\mu}(y)=\lim _{r \rightarrow 0} \hat{\mu}\left(B^{n-1}(y, r)\right) /\left(m_{n-1}\left(B^{n-1}(y, r)\right)\right.$ is the derivative of $\hat{\mu}$ with respect to the $m_{n-1}$ measure at $y \in \Omega^{\prime}$. 
Proof. By Lemma 5.1 for $m_{n-1}$-a.e. $y \in \Omega^{\prime}$,

$$
V\left(u, \gamma_{y}\right) \leq \liminf _{i \rightarrow \infty} \int_{\gamma} g_{i} d s
$$

whenever $\left(g_{i}\right)$ is a $B V_{A M}$ upper bound for $u$ in $B^{n-1}(z, r) \times(a, b), B^{n-1}(z, r) \subset \Omega^{\prime}$ and, by Lemma 5.2 , the Lebesgue theorem yields

$$
V\left(u, \gamma_{y}\right)=\lim _{r \rightarrow 0} m_{n-1}\left(B^{n-1}(y, r)\right)^{-1} \int_{B^{n-1}(y, r)} V\left(u, \gamma_{x}\right) d m_{n-1}(x)
$$

for $m_{n-1}$-a.e. $y \in \Omega^{\prime}$.

Fix a point $y \in \Omega^{\prime}$ such that (5.6) and (5.7) hold and the derivative $d \hat{\mu}(y)$ exists. Almost every point $y \in \Omega^{\prime}$ satisfies these conditions. Choose radii $r_{1}>r_{2}>\ldots$ with $\lim _{j \rightarrow \infty} r_{j}=0$, set $\left.B_{j}=B^{n-1}\left(y, r_{j}\right)\right)$ and for each $j$ pick a $B V_{A M}$ upper bound $\left(g_{i}^{j}\right)$ for $u$ in $B_{j} \times(a, b)$ such that

$$
\int_{B_{j} \times(a, b)} g_{i}^{j} d m_{n} \leq\left\|D_{B V} u\right\|\left(B_{j} \times(a, b)\right)+\frac{r_{j}^{n-1}}{2^{j}}
$$

for every $i=1,2, \ldots$. The Fatou lemma and (5.8) yield

$$
\begin{gathered}
V\left(u, \gamma_{y}\right)=\lim _{j \rightarrow \infty} m_{n-1}\left(B_{j}\right)^{-1} \int_{B_{j}} V\left(u, \gamma_{x}\right) d m_{n-1}(x) \\
\leq \liminf _{j \rightarrow \infty} m_{n-1}\left(B_{j}\right)^{-1} \int_{B_{j}}\left(\liminf _{i \rightarrow \infty} \int_{\gamma_{x}} g_{i}^{j} d s\right) d m_{n-1}(x) \\
\leq \liminf _{j \rightarrow \infty} m_{n-1}\left(B_{j}\right)^{-1} \liminf _{i \rightarrow \infty} \int_{B_{j} \times(a, b)} g_{i}^{j} d m_{n} \\
\leq \liminf _{j \rightarrow \infty} m_{n-1}\left(B_{j}\right)^{-1}\left(\left\|D_{B V} u\right\|\left(B_{j} \times(a, b)\right)+\frac{r_{j}^{n-1}}{2^{j}}\right)=d \hat{\mu}(y)
\end{gathered}
$$

and (5.5) follows.

The next theorem shows that the spaces $B V_{A M}(\Omega)$ and $B V(\Omega)$ defined in Section 3 and in (5.1), respectively, are the same.

Theorem 5.4. For each $u \in L^{1}(\Omega)$,

$$
\left\|D_{B V} u\right\|(\Omega) \leq\|D u\|(\Omega) \leq n\left\|D_{B V} u\right\|(\Omega) .
$$

Remark 5.5. It follows from the proof that the first inequality in (5.9) holds for all open sets $U \subset \Omega$.

Proof. Let $u \in B V(\Omega)$. By [7, 5.2.2, Theorem 2] there is a sequence of $C_{0}^{\infty}(\Omega)$ functions $u_{i}$ such that $u_{i} \rightarrow u$ in $L^{1}(\Omega)$ and

$$
\|D u\|(\Omega)=\lim _{i \rightarrow \infty} \int_{\Omega}\left|\nabla u_{i}\right| d m_{n} .
$$

Then passing to a subsequence (see Lemma 2.3) we can assume that the sequence $\left(\left|\nabla u_{i}\right|\right)$ is a $B V_{A M}$ upper bound for $u$ in $\Omega$. Hence

$$
\left\|D_{B V} u\right\|(\Omega) \leq \liminf _{i \rightarrow \infty} \int_{\Omega}\left|\nabla u_{i}\right| d m_{n}=\|D u\|(\Omega)
$$

and the first inequality in (5.9) follows. 
If $u \in B V(\Omega)$, then there exist measures $\left\|D_{j} u\right\|, j=1,2, \ldots, n$, defined on open sets $U \subset \Omega$ as

$$
\left\|D_{j} u\right\|(U)=\sup \left\{\int_{U} u \partial_{j} \varphi d m_{n}\right\}
$$

where the supremum is taken over all $\varphi \in C_{0}^{\infty}(U)$ such that $|\varphi| \leq 1$; see [7, 5.10]. By [7, the proof for Theorem 2 (7.10.2)] and Lemma [5.2] we have

$$
\left\|D_{n} u\right\|(\Omega)=\int_{\Omega^{\prime}} V\left(u, \gamma_{y}\right) d m_{n-1}(y) \leq\left\|D_{B V} u\right\|(\Omega)
$$

and a similar inequality holds for each $j=1,2, \ldots, n$. Let $\varepsilon>0$ and choose a function $\varphi=\left(\varphi_{1}, \varphi_{2}, \ldots, \varphi_{n}\right)$ in $C_{0}^{1}\left(\Omega, \mathbf{R}^{n}\right)$ such that $|\varphi| \leq 1$ and

$$
\|D u\|(\Omega) \leq \int_{\Omega} u \nabla \cdot \varphi d m_{n}+\varepsilon
$$

Now the above estimates yield

$$
\|D u\|(\Omega) \leq \sum_{j=1}^{n} \int_{\Omega} u \partial_{j} \varphi_{j} d m_{n}+\varepsilon \leq \sum_{j=1}^{n}\left\|D_{j} u\right\|(\Omega)+\varepsilon \leq n\left\|D_{B V} u\right\|(\Omega)+\varepsilon
$$

and letting $\varepsilon \rightarrow 0$ we obtain the second inequality in (5.9).

\section{REFERENCES}

[1] L. Ambrosio and S. Di Marino, Equivalent definitions of BV space and of total variation on metric measure spaces, J. Funct. Anal. 266 (2014), no. 7, 4150-4188, DOI 10.1016/j.jfa.2014.02.002. MR3170206

[2] L. Ambrosio, S. Di Marino, Weak and relaxed gradients for BV functions on metric measure spaces. To appear. Available at http://cvgmt.sns.it

[3] L. Ambrosio, S. Di Marino, and G. Savaré, On the duality between p-modulus and probability measures, J. Eur. Math. Soc. (JEMS) 17 (2015), no. 8, 1817-1853, DOI 10.4171/JEMS/546. MR.3372072

[4] L. Ambrosio, N. Gigli, and G. Savaré, Density of Lipschitz functions and equivalence of weak gradients in metric measure spaces, Rev. Mat. Iberoam. 29 (2013), no. 3, 969-996, DOI 10.4171/RMI/746. MR.3090143

[5] A. Björn and J. Björn, Nonlinear potential theory on metric spaces, EMS Tracts in Mathematics, vol. 17, European Mathematical Society (EMS), Zürich, 2011. MR2867756

[6] S. Di Marino, Recent advances in $B V$ and Sobolev spaces in metric measure spaces, PhD Thesis, Scuola Normale Superiore di Pisa, 2011-2014. Available at http://cvgmt.sns.it/media/doc/paper/2568/thesis final.pdf

[7] L. C. Evans and R. F. Gariepy, Measure theory and fine properties of functions, Studies in Advanced Mathematics, CRC Press, Boca Raton, FL, 1992. MR.1158660

[8] B. Fuglede, Extremal length and functional completion, Acta Math. 98 (1957), 171-219. MR.0097720

[9] E. De Giorgi, G. Letta, Una notion générale de convergence faible pour des fonctions croissantes d'ensemble, Ann. Scuola Norm. Sup. Pisa (1977), 61-99.

[10] J. Heinonen, Lectures on analysis on metric spaces, Universitext, Springer-Verlag, New York, 2001. MR 1800917

[11] J. Heinonen and P. Koskela, Quasiconformal maps in metric spaces with controlled geometry, Acta Math. 181 (1998), no. 1, 1-61, DOI 10.1007/BF02392747. MR.1654771

[12] R. Korte, P. Lahti, N. Shanmugalingam, Semmes family of curves and a characterization of functions of bounded variation in termes of curves, Manuscript.

[13] O. Martio, Functions of bounded variation and curves in metric measure spaces, to appear in Advances in Calculus of Variations. 
[14] M. Miranda Jr., Functions of bounded variation on "good" metric spaces (English, with English and French summaries), J. Math. Pures Appl. (9) 82 (2003), no. 8, 975-1004, DOI 10.1016/S0021-7824(03)00036-9. MR2005202

[15] N. Shanmugalingam, Newtonian spaces: an extension of Sobolev spaces to metric measure spaces, Rev. Mat. Iberoamericana 16 (2000), no. 2, 243-279, DOI 10.4171/RMI/275. MR.1809341

Department of Mathematics and Statistics, FI-00014 University of Helsinki, Finland

E-mail address: olli.martio@helsinki.fi 